\title{
Clinical and prognostic evaluation of patients admitted to the COVID-19 pandemic unit of the emergency department
}

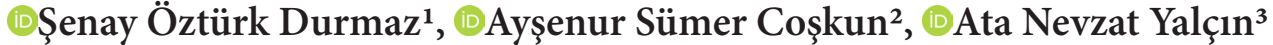 \\ ${ }^{1}$ Kepez State Hospital, Department of Infectious Diseases and Clinical Microbiology, Antalya, Turkey \\ ${ }^{2}$ Kepez State Hospital, Department of Anaesthesiology and Reanimation, Antalya, Turkey \\ ${ }^{3}$ Akdeniz University' Faculty of Medicine, Department of Infectious Diseases and Clinical Microbiology, Antalya, Turkey
}

Cite this article as: Öztürk Durmaz Ş, Sümer Coşkun A, Yalçın AN. Clinical and prognostic evaluation of patients admitted to the COVID-19 pandemic unit of the emergency department. J Health Sci Med 2021; 4(6): 835-839.

\begin{abstract}
Objective: This study aimed to evaluate to prognosis of patients admitted to the COVID-19 isolation unit of the emergency department and present epidemiological data by examining their demographic, clinical, laboratory and lung tomography findings.

Material and Method: A total of 504 patients presenting with COVID-19 symptoms were randomly and retrospectively evaluated based on electronic records obtained from the hospital archive.

Results: Of the patients, 216 (42.9\%) were female and 288 (57\%) were male. The RT-PCR test was positive in 291 (57.7\%) patients, and fever was the most common symptom in 280 (55.5\%). A total of 133 patients (26.4\%) were admitted to the inpatient ward. The WBC, Troponin-t, CRP, AST, ALT, LDH, D-dimer and ferritin levels were high and lymphocyte count was low in patients who were hospitalized. Sixty-one percent of the patients had a comorbid condition; $19 \%$ of had diabetes and $18.8 \%$ had hypertension as the most common underlying condition. Totally, $312(61.9 \%)$ had favipiravir, $106(21 \%)$ were favipiravir + hydroxychloroquine, 60 (11.9\%) used hydroxychloroquine alone, 26 (5.2\%) were followed up without treatment and to 147 of them, antibiotics were prescribed in addition to the medicine; $38.5 \%$ of the patients followed up in the intensive care unit were diabetic; 26 patients (5.2\%) were followed up in the intensive care unit. The mortality rate was found to be $1.9 \%$. At least one comorbid condition was present in all who were hospitalized and died in the intensive care unit.

Conclusion: The importance of a healthy diet and regular physical activity for metabolic conditions such as diabetes and hypertension as well as for fighting infections is well known. We consider that the mortality and morbidity rates due to the COVID-19 pandemic can be reduced by developing reliable and safe antiviral treatment options and implementing effective and fair vaccination policies.
\end{abstract}

Keywords: COVID-19, epidemiology, prognosis

\section{INTRODUCTION}

Coronavirus 2019 (COVID-19),caused by SARS-COV-2 was first reported in China at the end of 2019 and the World Health Organization (WHO) declared the disease as a pandemic on March 11, 2020. Since then, the number of cases has reached 102 million, and the number of those who died due to the virus has exceeded 2 million. The SARS-COV-2 infection can cause asymptomatic diseases, mild upper respiratory tract infections, respiratory failure, or severe viral pneumonia that may result in death (1-4). Although the real-time reverse transcriptase polymerase chain reaction (RT-PCR) test is the standard method used in the diagnosis of COVID-19, clinical, laboratory and thorax computed tomography (CT) evaluations also provide valuable data $(5-8)$.
In this study, we aimed to analyze the epidemiological data of patients admitted to the COVID-19 isolation unit of the emergency department by evaluating their clinical, laboratory, CT findings, comorbid conditions, and prognoses.

\section{MATERIAL AND METHOD}

The study was carried out with the permission of Health Science University, Antalya Training and Research Hospital Clinical Researchs Ethics Committee (Date: 01.04.2021, Decision No: 4-8). All procedures were carried out in accordance with the ethical rules and the principles of the Declaration of Helsinki. 
The study included a total of 504 patients who presented to the emergency department of our hospital with COVID-19 symptoms between June 1, 2020 and March 1, 2021. The patients' demographic data, medical histories, biochemical parameters including laboratory analysis, hemogram, $\mathrm{D}$-dimer, troponin- $\mathrm{T}$, $\mathrm{C}$ reactive protein (CRP) and ferritin, thorax (CT) findings, and treatment protocols recorded in the hospital's archive were electronically scanned in a retrospective manner.

Case definitions were made in line with the recommendations in with the current recommendations of the Turkish Ministry of Health COVID-19 Guidelines (9). Patients with positive SARS-COV-2 real-time reverse transcriptase polymerase chain reaction (RT-PCR) test results based on the respiratory tract sample wereaccepted as definitive COVID-19 cases. If the RT-PCR test was negative, but the patient had complaints related to the disease and findings compatible with viral pneumonia on CT scans, they were accepted as possible cases. Patients with a negative result from two consecutive PCR test performed at a 24-hour interval and normal CT findings were considered not to have COVID-19.

In the interpretation of the thoracic CT findings, the presence of ground-glass density of less than $3 \mathrm{~cm}$ in three or less foci was classified as mild pneumonia; consolidation or a ground-glass density of more than three foci or greater than $3 \mathrm{~cm}$ was classified as moderate pneumonia, and involvement of all lobes in both lungs and at least three lesions larger than $3 \mathrm{~cm}$ classified as severe pneumonia (10).

Conforming to the recommendations of the Turkish Ministry of Health COVID-19 Guidelines, the patients were hospitalized based on the following indications: poor prognosis markers; comorbid conditions, such as hypertension (HT), diabetes mellitus, chronic lung disease (lymphocyte number $<850 \backslash \mathrm{mm} 2$, ferritin $>500 \mathrm{ng} \backslash \mathrm{mL}$, $\mathrm{D}$-dimer $>1000 \mathrm{ng} \backslash \mathrm{mL}$ ) or suspicion of the severe form of the disease (9). Medications to be given to the patients, their doses, and treatment options were arranged in line with the recommendations of the same Guidelines (9).

All statistical analyses were performed using IBM SPSS version 25.0 (SPSS Inc., Chicago, Illinois, USA). Tables are presented as Mean $\pm \mathrm{SD}$ for continuous variables, while categorical variables are presented as number $(\mathrm{N})$ and percentage (\%). Comparisons between groups were made using the independent t-test and ANOVA test for continuous variables. Among the Post-Hoc tests, Turkey test was utilized. Chi-square test was applied to compare categorical variables. Pearson correlation analysis was used for the relations of continuous variables with each other and $\mathrm{p}<0.005$ was considered statistically significant.

\section{RESULTS}

Demographic data of patients given in Table 1. Among 504 patients who presented to the COVID-19 unit of the emergency department, fever was the most common symptom ( $\mathrm{n}=280,55.5 \%)$. Respectively, weakness and malaise in 232 patients (46\%), cough in 120 patients (23.8\%), muscle-joint pain in 98 patients (19.4\%), shortness of breath in 88 patients $(17.4 \%)$, sore throat in 76 patients (15\%), headache in 76 patients $(15 \%)$, loss of taste and smell in 43 patients (8.5\%), chest and back pain in 37 patients (7.3\%), diarrhea in 24 patients (4.8\%), anorexia in 12 patients (2.4\%), abdominal pain and nausea in 6 patients (1.2\%) were observed.

\begin{tabular}{|c|c|c|}
\hline $\begin{array}{l}\text { Demographic } \\
\text { Variables }\end{array}$ & $\begin{array}{c}\mathrm{N} \text { and/or XOR } \\
(\min -\mathrm{max})\end{array}$ & $\begin{array}{c}\% \text { and/or Mean } \\
\pm \text { SD }\end{array}$ \\
\hline \multicolumn{3}{|l|}{ Gender } \\
\hline Female & 216 & 42.9 \\
\hline Male & 288 & 57.1 \\
\hline Age (years) & $46.00(18-105)$ & $46.46 \pm 17.57$ \\
\hline \multicolumn{3}{|l|}{ PCR } \\
\hline Positive & 291 & 57.7 \\
\hline Negative & 121 & 24.0 \\
\hline Possible Case & 92 & 18.3 \\
\hline \multicolumn{3}{|c|}{ Tomography (Pneumonia findings) } \\
\hline Doesn't exist & 211 & 41.8 \\
\hline Mild & 227 & 45.1 \\
\hline Moderate & 53 & 10.5 \\
\hline Severe & 13 & 2.6 \\
\hline \multicolumn{3}{|l|}{ Hospital Stay (day) } \\
\hline $1-7$ & 75 & 60.0 \\
\hline $8-14$ & 45 & 36.0 \\
\hline$>14$ & 5 & 4.0 \\
\hline \multicolumn{3}{|c|}{ Treatment Conditions } \\
\hline Outpatient & 371 & 73.6 \\
\hline $\begin{array}{l}\text { Inpatients (ICU + } \\
\text { ward) }\end{array}$ & 133 & 26.4 \\
\hline $\begin{array}{l}\text { ICU hospitalization } \\
\text { (day) }\end{array}$ & $8.50(1.00-40.00)$ & $11.34 \pm 10.69$ \\
\hline LYM & $16000.0(14.60-150000.0)$ & $1786.43 \pm 1101.16$ \\
\hline Trop T & $4.85(2.00-1280.00)$ & $12.88 \pm 63.81$ \\
\hline WBC & $6535.00(500.0-50000.0)$ & $7806.84 \pm 4520.03$ \\
\hline PLT & $216.00(4.12-206000.0)$ & $1057.34 \pm 12746.73$ \\
\hline CRP & $8.90(0.10-334.00)$ & $33.19 \pm 53.27$ \\
\hline AST & $22.00(4.00-299.00)$ & $29.16 \pm 29.59$ \\
\hline ALT & $22.00(1.00-804.00)$ & $33.04 \pm 56.55$ \\
\hline $\mathrm{LDH}$ & $204.50(18.80-2650.00)$ & $235.02 \pm 153.65$ \\
\hline D-dimer & $149.00(10.80-24000.00)$ & $360.10 \pm 1357.69$ \\
\hline Ferritin & $97.00(1.00-10000.00)$ & $216.19 \pm 663.44$ \\
\hline \multicolumn{3}{|l|}{ Rate of Death } \\
\hline Alive & 494 & 98.0 \\
\hline Dead & 10 & 2.0 \\
\hline
\end{tabular}

For treatment, favipiravir was used in 312 of the patients, favipiravir+hydroxychloroquine in 106, hydroxychloroquine alone in 60 , while 26 patients were followed up without treatment, and 147 were additionally prescribed antibiotics. 
A statistically significant difference was observed between outpatients and hospitalized patients in terms of age. The mean age of the patients admitted to the was higher compared to the outpatients $(\mathrm{p}<0.001)$. The remaining parameters that showed statistically significant differences between the outpatient and inpatient groups were lymphocyte (LYM) count, troponin-T, leukocyte (WBC), C- reactive protein (CRP), aspartate aminotransferase (AST), alanine aminotransferase (ALT), lactic asid dehydrogenase (LDH), D-dimer, and ferritin. The LYM values of the outpatients were significantly higher compared to the hospitalized patients $(\mathrm{p}<0.001)$. The troponin- $\mathrm{T}$ of the hospitalized patients were higher compared to the outpatients $(\mathrm{p}=0.023)$. The WBC values of hospitalized patients were found to be higher than outpatients $(\mathrm{p}=0.049)$. The troponin-T, WBC, CRP, AST, ALT, LDH, $\mathrm{D}$-dimer and ferritin values of the hospitalized patients were significantly higher compared to the outpatients $(\mathrm{p}=0.023, \mathrm{p}=0.049, \mathrm{p}<0.001, \mathrm{p}<0.001, \mathrm{p}=0.029, \mathrm{p}<0.001$, $\mathrm{p}=0.021$, and $\mathrm{p}=0.002$, respectively) (Table 2 ). There was no statistically significant difference in age between the male and female patients and between the patients with different hospitalization days.

Table 2. Comparison of various variables between inpatients and outpatients

\begin{tabular}{|c|c|c|c|}
\hline Variables & $\begin{array}{c}\text { Outpatients } \\
(n=371) \text { Mean } \pm \text { SD }\end{array}$ & $\begin{array}{c}\text { Inpatients } \\
(n=133) \text { Mean } \pm S D\end{array}$ & $\mathbf{P}$ \\
\hline Age & $42.21 \pm 15.20$ & $57.86 \pm 18.44$ & $<0.001^{* *}$ \\
\hline LYM & $1924.61 \pm 1182.23$ & $1413.55 \pm 726.42$ & $<0.001^{* *}$ \\
\hline Trop-T & $6.55 \pm 17.96$ & $29.92 \pm 117.59$ & $0.023^{\star}$ \\
\hline WBC & $7554.35 \pm 4385.70$ & $8483.19 \pm 4813.25$ & $0.049^{*}$ \\
\hline PLT & $1325.57 \pm 14914.33$ & $338.81 \pm 1265.60$ & 0.440 \\
\hline CRP & $19.24 \pm 34.91$ & $70.58 \pm 72.67$ & $<0.001^{\star *}$ \\
\hline AST & $24.23 \pm 15.69$ & $42.35 \pm 48.32$ & $<0.001^{\star \star}$ \\
\hline ALT & $27.94 \pm 27.30$ & $46.69 \pm 97.80$ & $0.029^{*}$ \\
\hline LDH & $218.76 \pm 165.76$ & $278.79 \pm 103.33$ & $<0.001^{\star \star}$ \\
\hline D-dimer & $230.36 \pm 619.92$ & $710.20 \pm 2375.57$ & $0.021^{*}$ \\
\hline Ferritin & $128.26 \pm 173.24$ & $451.10 \pm 1210.51$ & $0.002^{\star}$ \\
\hline \multicolumn{4}{|c|}{$\begin{array}{l}\text { Independent T-Test, }{ }^{* *} \mathrm{p}<0.001,{ }^{*} \mathrm{p}<0.005 \\
\text { Lymphocyte (LYM) count, troponin-T, leukocyte (WBC), platelet count (PLT), C- } \\
\text { reactive protein (CRP), aspartate aminotransferase (AST), alanine aminotransferase } \\
\text { (ALT), lactic asid dehydrogenase (LDH) }\end{array}$} \\
\hline
\end{tabular}

Of the total 504 patients who presented to the emergency department with COVID-19, 96 (19\%) were diabetic. Twelve patients were healthcare workers (2.38\%) and 15 (3\%) were pregnant. Among the inpatients, 63 (47.36\%) had diabetes (Table 3).

The rate of admission to intensive care was $5.2 \%$. The mortality rate was found to be $1.9 \%$. All patients hospitalized in the intensive care unit had at least one comorbid condition.

\begin{tabular}{|lc|}
\hline \multicolumn{2}{|l|}{ Table 3. Comorbidity distributions of 504 patients } \\
\hline Comorbidity & N (\%) \\
\hline Diabetes & $96(19)$ \\
Hypertension & $95(18.8)$ \\
CVD & $53(10.5)$ \\
Chronic lung disease & $24(4.7)$ \\
Chronic neurological disease & $12(2.7)$ \\
Malignancy & $11(2.6)$ \\
Chronic kidney disease & $10(2.4)$ \\
Chronic liver disease & $8(2)$ \\
Pregnant & $15(3)$ \\
\hline CVD: Cardio-Vascular Disease (Coronary Artery Disease, Ischemic Heart Disease, \\
Congestive Heart Failure)
\end{tabular}

Of the 26 patients that received intensive care, 10 had CVD + Diabetes, four had hypertension, two had CVD + malignancy, two had CVD + Chronic Respiratory disease, one had chronic neurological disease, one had immunosuppressed + CVD, one had chronic neurological disease + chronic respiratory tract disease, one had chronic kidney disease + chronic neurological disease $+\mathrm{CVD}$, and one had malignancy.

Among these 26 patients, 10 (three female and seven male) died, and six had CVD + diabetes, two had chronic neurological disease + chronic respiratory disease, two had CVD + chronic respiratory disease + chronic liver disease.

Lastly, of the 133 people hospitalized in the service, 18 were transferred from the service to intensive care, and 8 were directly admitted to intensive care from the emergency service.

\section{DISCUSSION}

There is no specific clinical feature that can reliably distinguish COVID-19 from other respiratory viral infections. COVID-19 has a wide range of manifestations from asymptomatic or mild pneumonia to acute respiratory failure and death.

In our study, the average age of all patients was 46.56 and male patients were in the majority (57\%) were male, which is in agreement with the results of previous studies $(3,11)$. WHO has defined common COVID-19 symptoms as fever, fatigue and dry cough. In addition, shortness of breath, myalgia, sore throat and diarrhea have been reported in very few patients (12). In our study, the rate of RT-PCR positivity was found to be $57.7 \%$. Similarly, in another study conducted in Turkey Karakoç et al. (13) reported the rate of PCR positivity as $65.3 \%$. In the current study, 280 patients (55\%) presented with fever, 232 (46\%) with weakness and malaise, 120 (23\%) with dry cough, 98 (19.4\%) with muscle-joint pain, 88 (17.4\%) with shortness of breath, 76 (15\%) with sore throat, 76 (15\%) with headache, $43(\% 8.5)$ loss of taste and smell, $37(\% 7.3)$ with chest and back pain, 24 (4.8\%) with diarrhea, 12 (2.4\%) with anorexia, and six (1.2\%) with 
abdominal pain and nausea. This clinical spectrum was similar to most other studies (3,14-16).

According to the COVID-19 Guidelines of the Turkey's Ministry of Health Public Health General Directorate, the disease is classified as uncomplicated, pneumonia and severe pneumonia. Patients without fever, muscle-joint pain, cough, sore throat, nasal congestion, respiratory distress, tachypnea and $\mathrm{SpO} 2<93 \%$, with no underlying diseases (cardiovascular diseases, diabetes mellitus, hypertension, cancer, chronic lung diseases along with other immunosuppressive conditions), those under 50 years of age, those with normal chest radiography or CT findings, and those without poor prognostic values in blood tests performed at admission (blood lymphocyte count $<800 / \mu \mathrm{l}$ or CRP $>40 \mathrm{mg} / \mathrm{L}$ or ferritin $>500 \mathrm{ng} /$ $\mathrm{mL}$ or $\mathrm{D}$-dimer $>1000 \mathrm{ng} / \mathrm{mL}$ etc.) are defined as uncomplicated diseases, while patients with a respiratory rate of less than 30 per minute are evaluated in the mild pneumonia category, and those with a $\mathrm{SpO} 2$ level below $90 \%$ in room air, bilateral diffuse pneumonia on chest X-ray or tomography, and poor prognostic values in blood tests at the time of admission are classified as severe pneumonia (17).

The severity of COVID-19 and its mortality course have been shown to be associated with cardiovascular diseases, diabetes, hypertension, chronic lung and kidney diseases, and cancers (14). The fatality rate is higher among those with comorbid conditions. According to the data reported from China, the rate of mortality was $10.5 \%$ in patients with cardiovascular diseases, $7.3 \%$ in the presence of diabetes, $6.3 \%$ in the presence of chronic respiratory failure, $6.0 \%$ in the presence of hypertension, and $5.6 \%$ in the presence of cancer (18-19). When we evaluated the 504 patients included in our study, those with comorbidities constituted $61 \%$ of the whole sample. The most common comorbidities were diabetes (19\%) and hypertension (18.8\%), followed by CVD (10.5\%), chronic lung disease (4.7\%), chronic neurological disease $(2.7 \%)$, and malignancy (2.6\%). While the mortality rate was $0.9 \%$ among the patients without any comorbidity, it was much higher inthose with comrbidities. In a previous study, the rate of mortality was reported to be $7.4 \%$ in patients with diabetes (20). In another study, 32.3\% $(10 / 31)$ of patients that required ICU were diabetic, while the rate of diabetics among those that did not require ICU hospitalization was determined as $9.2 \%$ (22). Similarly, in our study 10 of the 26 (38.5\%) patients followed up in the intensive care unit were diabetic.

In our study, at least one comorbid condition was present among the 26 patients admitted to the intensive care unit and all 10 patients who died. Of the 10 patients who died, three were female and seven were male, and six had CVD + diabetes, two had chronic neurological disease
+ chronic respiratory disease $+\mathrm{HT}$, and two had CVD + chronic respiratory disease + chronic liver disease. In a case group consisting of 355 patients who died due to COVID-19 in Italy, the presence of underlying chronic diseases was emphasized in all except 3 cases (21). Similarly, in our study, at least one comorbid condition was found in all patients who died.

Since there is no standard treatment regimen for COVID-19, there are differences between countries. For the treatment planning of symptomatic patients, we followed the guidelines of the Turkish Ministry of Health to decide whether they required inpatient or outpatient treatment. In the treatment of our patients, we used favipiravir in 312 (61.9\%), favipiravir + hydroxychloroquine 106 (21\%), and hydroxychloroquine alone in $60(11.9 \%)$, while we followed up 26 patients without treatment and prescribed additional antibiotics for 147 patients. Chloroquine is a widely used malaria drug that was shown to have potential broad-spectrum antiviral properties in 2006. It has been reported to successfully block SARS-CoV-2 infections at a low concentration. Favipiravir is a new RNA-dependent RNA polymerase (RdRp) inhibitor. In addition to its antiinfluenza activity, favipiravir has the ability to inhibit the replication of flavi-, alpha, filo-, bunya-, arena-, neuro-, and other RNA viruses. Therefore, it may have a potential antiviral effect against SARS-CoV-2, which is an RNA $\operatorname{virus}(22,23)$.

All the patients who died in our study had the severe form of the disease and required intensive care. In Turkey, the rate of cases diagnosedwith severe COVID-19 is similar to China, and $6 \%$ of the total cases detected are followed up in the ICU (24). Similarly, in our study, the rate of patients followed up in the ICU was $5.2 \%$. According to the WHO statement on March 3, 2020, the global fatality rate due to COVID-19 is 3.4\% (25). In our study, the mortality rate was found to be $1.9 \%$. In Turkey, the population over the age of 65 years constitutes about $9 \%$ of the total population in contrast to $20 \%$ in many European countries. The younger population and treatment capacity not being exceeded may be among the reasons for the lower mortality rate observed in our country $(26,27)$.

The small number of patients and limited treatment options, single-center study and retrospective can be considered as the limitations of our study.

In our study, $47.36 \%$ of hospitalized patients had diabetes. This indicates the importance of blood glucose regulation in preventing mortality-morbidity due to COVID-19. The mortality rate among our patients was as low as $1.9 \%$, which shows the importance and success of the Turkish health system infrastructure in combating epidemic diseases. 


\section{CONCLUSION}

In the current study, diabetes, hypertansion and CVD, were the most commonly observed coöorbidities among the patients with COVID-19 that required hospitalization or intensive care. We consider that these health problems can be managed in most people by a healthy diet and regular physical activity. The challenging process created by the COVID-19 pandemic has once again emphasized the importance of nutrition. We believe that the mortality and morbidity caused by the pandemic can only be reduced with the scientific world developing reliable and safe antiviral treatment options and implementing effective and fair vaccination policies.

\section{ETHICAL DECLARATIONS}

Ethics Committee Approval: The study was carried out with the permission of Health Science University, Antalya Training and Researchs Hospital Clinical Research Ethics Committee (Date: 01.04.2021, Decision No: 4-8).

Informed Consent: Because the study was designed retrospectively, no written informed consent form was obtained from patients.

Referee Evaluation Process: Externally peer-reviewed.

Conflict of Interest Statement: The authors have no conflicts of interest to declare.

Financial Disclosure: The authors declared that this study has received no financial support.

Author Contributions: All of the authors declare that they have all participated in the design, execution, and analysis of the paper, and that they have approved the final version.

\section{REFERENCES}

1. Hasöksüz M, Kiliç S, Saraç F. Coronaviruses and sars-cov-2. Turk J Med Sci 2020; 50: 549-56.

2. Lai CC, Shih TP, Ko WC, Tang HJ, Hsueh PR. Severe acute respiratory syndrome coronavirus 2 (SARS-CoV-2) and coronavirus disease-2019 (COVID-19): The epidemic and the challenges. Int J Antimicrob Agents 2020; 55: 105924.

3. Guan WJ, Ni ZY, Hu Y, et al. Clinical characteristics of coronavirus disease 2019 in China. New Engl J Med 2020; 382: 1708-20.

4. Salehi S, Abedi A, Balakrishnan S, Gholamrezanezhad A. Coronavirus disease 2019 (COVID-19): a systematic review of imaging findings in 919 patients. Am J Roentgenol 2020; 215: 87-93.

5. Li Z, Yi Y, Luo X, et al. Development and clinical application of a rapid IgM-IgG combined antibody test for SARS-CoV-2 infection diagnosis. J Med Virol 2020; 92: 1518-24.

6. Hao W, Li M. Clinical diagnostic value of $\mathrm{CT}$ imaging in COVID-19 with multiple negative RT-PCR testing. Travel Med Infect Dis 2020; 34: 101607.

7. Wang S, Kang B, Ma J, et al. A deep learning algorithm using CT images to screen for corona virus disease (COVID-19). Eur Radiol 2021: 1-9.

8. Zhai P, Ding Y, Wu X, Long J, Zhong Y, Li Y. The epidemiology, diagnosis and treatment of COVID-19. Int J Antimicrob Agents 2020; 55: 105955.
9. COVID-19 Information Page. Access date: 11, 2020. Access: https://covid19.saglik.gov.tr/TR 66935/genelkoronavirus-tablosu. html

10. British Society of Thoracic Imaging. Thoracic imaging in COVID-19 infection. Guidance for the reporting radiologist. Version 2. 16 March 2020 [Internet]. UK BSTI [Access 15 June 2020].https: \|www.bsti.org.uk\media $\backslash$ resources $\backslash$ files $\backslash$ BSTI_ COVID-19_Radiology_Guidance_version_2_16.03.20.pdf.

11.Zhang JJ, Dong X, Cao YY, et al. Clinical characteristics of 140 patients infected with SARS-CoV-2 in Wuhan, China. Allergy 2020; 75: 1730-41.

12. World Health Organization. (2020). Coronavirus disease 2019 (COVID-19): situation report, 60. https://www.who.int/healthtopics/coronavirus\#tab=- tab_3. Access date 01.07.2020.

13. Karakoc ZC, Pinarbasi Simsek B, Asil R, et al. First wave in the COVID-19 pandemic: one center experience. Klimik J/Klimik Derg 202; 33: 3.

14.Zhou F, Yu T, Du R, et al. Clinical course and risk factors for mortality of adult inpatients with COVID-19 in Wuhan, China: a retrospective cohort study. The Lancet 2020; 395: 1054-62.

15. Elshafeey F, Magdi R, Hindi N, et al. A systematic scoping review of COVID-19 during pregnancy and childbirth. Int J Gynecol Obstet 2020; 150: 47-52.

16. Wang $\mathrm{D}, \mathrm{Hu} \mathrm{B}, \mathrm{Hu} \mathrm{C}$, et al. Clinical characteristics of 138 hospitalized patients with 2019 novel coronavirus-infected pneumonia in Wuhan, China. JAMA 2020; 323: 1061-9.

17. Turkey Health Ministry (2020). General Directorate of Public Health, Covid-19 (SARS-CoV-2) guide (Scientific Committee Study). Access date:10 July 2020. Available from: https:\\hsgm. saglik.gov.tr $\backslash$ tr $\backslash$ covid 19.

18. Wu Z, McGoogan JM. Characteristics of and important lessons from the coronavirus disease 2019 (COVID-19) outbreak in China: summary of a report of 72314 cases from the Chinese Center for Disease Control and Prevention. JAMA 2020; 323: 1239-42.

19. Yang JK, Feng Y, Yuan MY, et al. Plasma glucose levels and diabetes are independent predictors for mortality and morbidity in patients with SARS. Diabetic Med 2006; 23: 623-8.

20. Allard R, Leclerc P, Tremblay C, Tannenbaum TN. Diabetes and the severity of pandemic influenza A (H1N1) infection. Diabetes Care 2010; 33: 1491-3.

21.Grasselli G, Pesenti A, Cecconi M. Critical care utilization for the COVID-19 outbreak in Lombardy, Italy: early experience and forecast during an emergency response. JAMA 2020; 323: 1545-6.

22. Wang M, Cao R, Zhang L, et al. Remdesivir and chloroquine effectively inhibit the recently emerged novel coronavirus (2019$\mathrm{nCoV}$ ) in vitro. Cell Res 2020; 30: 269-71.

23. Delang L, Abdelnabi R, Neyts J. Favipiravir as a potential countermeasure against neglected and emerging RNA viruses. Antivir Res 2018; 153: 85-94.

24. Turkey Health Ministry (2020). The current situation in Turkey. 2020. Available from https:I|saglik.gov.tr\Access date: June 1, 2020 .

25. World Health Organization. (2020). WHO Director-General's opening remarks at the media briefing on COVID-19-11 March 2020 .

26. Eraksoy, H. COVID-19 Pandemic: Leaving First Four Months Behind/ COVID-19 Pandemic. KLIMIK J 2020; 33: 1-2.

27.Genç A, Kurtaiş Aytür Y. Acute and Post-acute pulmonary rehabilitation in COVID-19 infection. COVID-19 Pandemisi ve Fiziksel Tip ve Rehabilitasyon. 1st Edition. Ankara: Türkiye Klinikleri; 2020; 7-12. 